\title{
Challenging Nuclear Cardiology Research: Stimulating Discovery, Validation, and Clinical Relevance
}

\author{
Vasken Dilsizian \\ University of Maryland School of Medicine, Baltimore, Maryland
}

$\mathbf{M}$ ost of what we know of nuclear cardiology has been discovered in the last nearly $50 \mathrm{y}$. In tracing the history of nuclear cardiology research, we find that, early on, it attracted the attention of some of the best minds in nuclear medicine and cardiology, who were working closely with highly talented, skilled, and academically engaged radiochemists, physicists, and technologists. This successful blueprint of an integrated approach to scientific discovery that addresses clinically relevant issues remains true today and should remain true in the future.

Nuclear imaging expands the traditional imaging of anatomic structures to physiologic and biochemical structures or biologic processes. A key attribute of nuclear molecular imaging is that it allows clinicians to visualize cellular functions that influence progression of disease, therapeutic responsiveness, and ultimately patient outcome. Such molecularly targeted imaging has the potential to direct new drug development and new gene- and cellbased therapies and to determine the subset of patients who are most likely to respond to such therapies. Since the introduction of myocardial perfusion (1) and metabolic agents (2), there has been explosive growth in the literature on, and National Institutes of Health funding of, experimental studies on cardiovascular molecular imaging. Unfortunately, such advances in the basic sciences have not translated into clinically approved diagnostic or therapeutic agents. Important challenges that have limited the progression to clinical reality include cost, scalability, and regulatory burden (3).

\section{HOW TO INCREASE THE RELEVANCE OF RESEARCH}

For a productive pursuit of scientific investigation, it is essential that a lead or idea be clinically relevant and that it address an unmet need. Next, there must be methodologic and imaging procedures capable of visually or quantitatively capturing the specific biologic process of interest, such as metabolic pathway, receptor biology, or enzyme regulation. For example, the ability to image the metabolic shift of energy production from fatty acids to glucose in the setting of reduced myocardial blood flow at rest has helped explain the pathophysiology of viable, hibernating myocardium and critical patient management decisions regarding coronary artery revascularization, left ventricular assist device placement, cardiac transplantation, or continued medical therapy $(4,5)$.

Received Nov. 2, 2017; revision accepted Nov. 6, 2017.

For correspondence or reprints contact: Vasken Dilsizian, Department of Diagnostic Radiology and Nuclear Medicine, University of Maryland Medical Center, 22 S. Greene St., Room N2W78, Baltimore, MD 21201.

E-mail: vdilsizian@umm.edu

Published online Nov. 16, 2017.

COPYRIGHT (c) 2018 by the Society of Nuclear Medicine and Molecular Imaging. DOI: 10.2967/jnumed.117.203042
In medicine, we must try to infer the nature of the biologic system from measured dynamic function and to derive information about their causes and interrelations in order to understand normal and abnormal disease conditions. Dynamic and quantitative analysis is a key virtue of nuclear radiotracer-based imaging. It allows assessment of changes in enzymatic or receptor activity as a function of disease severity and time. Creation of the time profile of the change in disease process is critical in following progression or regression of disease and the therapeutic effect of medications over a long period, such as days, weeks, or months. The latter also has implications for decreasing the number of animals sacrificed for the purposes of scientific investigation.

In this context, it is important to identify the best investigational tools (radiotracers and imaging systems) for detecting and localizing the most complex physiologic processes. Radiotracer-based approaches toward noninvasive cardiovascular imaging have the advantage of targeting small and sparse biologically relevant processes with high selectivity and sensitivity. When coupled with the favorable high resolution of CT and MRI, these approaches allow anatomic colocalization of the underlying pathophysiologic mechanisms responsible for diseases of the myocardium and vasculature (6). The development of such hybrid or multimodality imaging has facilitated validation of specific molecular signals and optimized targeted imaging.

\section{NOVEL FINDINGS AND APPLICATIONS: A TALE OF 3 RADIOTRACERS}

The greatest interest in the field lies in unique and original discoveries of radiotracers or in novel applications of previously approved radiotracers. Below are representative radiotracers that fulfill the innovation criterion.

${ }^{123}$ I-metaiodobenzylguanidine, a sympathetic neurotransmitter analog of norepinephrine, represents one of the few molecular imaging agents that can serve as a model for enduring the bench-to-bedside scientific journey of receiving U.S. Food and Drug Administration (FDA) approval for imaging neuroendocrine tumors and subsequently fulfilling the criterion of novel application for cardiac neuronal imaging for risk evaluation in heart failure $(7,8)$. A current multicenter clinical trial is investigating whether ${ }^{123}$ I-metaiodobenzylguanidine may also help identify patients who are at highest risk for sudden death and in whom automatic implantable cardiac devices will have the greatest cost effectiveness and clinical impact.

99m Tc-pyrophosphate, an FDA-approved bone-seeking radiotracer that has been used by nuclear cardiologists for cardiac infarction imaging, now provides the opportunity to diagnose cardiac amyloidosis and to differentiate light-chain amyloidosis from transthyretin-related cardiac amyloidosis (9). The diagnosis 
of cardiac amyloidosis is challenging because the clinical manifestations of cardiac amyloidosis overlap other heart diseases, such as heart failure with preserved ejection fraction, and are often not recognized until late stages of the disease. The gold standard for diagnosing cardiac amyloidosis is an endomyocardial biopsy, which is invasive and carries a low but substantial risk of heart perforation. ${ }^{99 m}$ Tc-pyrophosphate imaging provides the opportunity for early noninvasive diagnosis of cardiac amyloidosis, differentiating lightchain amyloidosis from transthyretin-related cardiac amyloidosis, monitoring disease progression, and assessing treatment response.

Another potentially unique radiotracer with novel clinical application is a methyl branched-chain fatty acid, $\beta$-methyl- $p-{ }^{123} \mathrm{I}-$ iodophenyl-pentadecanoic acid ( ${ }^{123} \mathrm{I}$-BMIPP). Delayed recovery of regional fatty acid metabolism, as an imprint of a prior ischemic event, has been shown with ${ }^{123}$ I-BMIPP and termed ischemic memory $(10,11)$. In an open-label phase IIA clinical trial, the utility of fatty acid metabolism with ${ }^{123} \mathrm{I}$-BMIPP to detect myocardial ischemic memory was shown up to $30 \mathrm{~h}$ after exercise-induced ischemia (10). In a subsequent phase III clinical trial, the safety and efficacy of ${ }^{123} \mathrm{I}-$ BMIPP were evaluated in patients presenting to an emergency department with symptoms suggestive of acute coronary syndrome (12). When the ${ }^{123}$ I-BMIPP data were added to the initial clinical diagnosis alone, the sensitivity for the acute coronary syndrome diagnosis nearly doubled without sacrificing specificity. Not only did the use of ${ }^{123} \mathrm{I}-$ BMIPP imaging add incremental value toward early diagnosis of acute coronary syndrome, but it substantially extended the time window within which noninvasive imaging may assist in the evaluation of patients with antecedent ischemia and suspected acute coronary syndrome beyond the resolution of symptoms. However, despite the salutary phase II and III clinical trial results, ${ }^{123}$ I-BMIPP is not FDA-approved in the United States, because the parent company that had the U.S. patent for ${ }^{123}$ I-BMIPP was acquired by a pharmaceutical company whose focus and priority were to expand its oncology pipeline rather than cardiology. ${ }^{123}$ I-BMIPP has been commercially available and clinically used in Japan for more than $20 \mathrm{y}$.

\section{POSSIBILITIES FOR FUTURE DISCOVERIES}

Nuclear cardiology research offers possibly the clearest insight into the unsolved mechanisms for disease processes, areas of controversy, and promising areas of future investigation on molecular imaging. Disagreement and controversy in the literature often increase rather than decrease interest in the field. Certain areas of investigation may not necessarily be controversial but rather a blank canvas in the sense that explanations are needed through experimental and translational research.

Judging by the rich history of the field of nuclear cardiology and its present status, many future discoveries even more exciting than the past ones will emerge from the experimental and translational use of these radiotracers. However, innovative mechanisms would need to be worked out to facilitate more cost-effective and rapid translation from experimental animals to large animals and humans, with the support of the National Institutes of Health and the FDA as potential partners in this translational journey. This impasse also requires academia-industry partnerships, where the industry partner has to be confident that the investment in time and resources for the potential diagnostic imaging agent is justifiable.

\section{BARRIERS TO ACADEMIC PURSUIT FOR EARLY-CAREER PHYSICIANS}

Another barrier to innovative nuclear cardiology research is the limited number of training programs that have the expertise and requisite facilities to perform high-quality, molecularly based, translational imaging studies and train the next generation of investigators. There is a need for a concerted effort to promote mentoring and establish philanthropic foundations that will fund research. Although the National Institutes of Health has increased funding of career development grants, unfortunately, only a small number of early-career physicians have benefited, because funding of the entire cohort has decreased (13). Academic centers and institutions need to embrace such early-career physicians and decrease the pressure to meet clinical relative-value-unit goals and provide credit for academic pursuits such as grant writing, research, and teaching.

Finally, we cannot forget that outreach and partnering with professional societies such as the American College of Cardiology, American Heart Association, and American Society of Nuclear Cardiology, along with education and awareness of radiotracer-based targeted molecular imaging through imaging journals (e.g., The Journal of Nuclear Medicine and Journal of Nuclear Cardiology) and annual scientific sessions, are all requisites for stimulating the discovery, validation, and clinical relevance of nuclear cardiology research.

\section{DISCLOSURE}

No potential conflict of interest relevant to this article was reported.

\section{REFERENCES}

1. Dilsizian V, Taillefer R. Journey in evolution of nuclear cardiology: will there be another quantum leap with the F-18 labeled myocardial perfusion tracers? JACC Cardiovasc Imaging. 2012;5:1269-1284.

2. Gewirtz H, Dilsizian V. Myocardial viability: survival mechanisms and molecular imaging targets in acute and chronic ischemia. Circ Res. 2017;120:11971212 .

3. Buxton DB, Antman M, Danthi N, et al. Report of the National Heart, Lung, and Blood Institute working group on the translation of cardiovascular molecular imaging circulation. Circulation. 2011;123:2157-2163.

4. Dilsizian V, Bacharach SL, Beanlands RS, et al. ASNC imaging guidelines/ SNMMI procedure standard for positron emission tomography (PET) nuclear cardiology procedures. J Nucl Cardiol. 2016;23:1187-1226.

5. Dilsizian V. Highlights from the updated joint ASNC/SNMMI PET myocardial perfusion and metabolism clinical imaging guidelines. J Nucl Med. 2016;57: 1327-1328.

6. Tian J, Smith M, Ahmad G, et al. Integration of three dimensional scar maps using single photon emission computed tomography to guide ventricular tachycardia ablation. J Nucl Med. 2012;53:894-901.

7. Jacobson AF, Senior R, Cerqueira MD, et al. Myocardial iodine-123 metaiodobenzylguanidine imaging and cardiac events in heart failure: results of the prospective ADMIRE-HF (AdreView Myocardial Imaging for Risk Evaluation in Heart Failure) study. J Am Coll Cardiol. 2010;55:2212-2221.

8. Dilsizian V, Eckelman WC. Myocardial blood flow and innervation measures from a single scan: an appealing concept but a challenging paradigm. J Nucl Med. 2015;56:1645-1646.

9. Dilsizian V. Greatest opportunities for growth in nuclear cardiology. $\mathrm{J} \mathrm{Nucl}$ Cardiol. 2017;24:1119-1120.

10. Dilsizian V, Bateman TM, Bergmann SR, et al. Metabolic imaging with $\beta$-methyl-p-[ $\left.{ }^{123} \mathrm{I}\right]$-iodophenyl-pentadecanoic acid (BMIPP) identifies ischemic memory following demand ischemia. Circulation. 2005;112:2169-2174.

11. Taegtmeyer H, Dilsizian V. Imaging myocardial metabolism and ischemic memory. Nat Clin Pract Cardiovasc Med. 2008;5(suppl):S42-S48.

12. Kontos MC, Dilsizian V, Weiland F, et al. Iodofiltic acid I 123 (BMIPP) fatty acid imaging improves initial diagnosis in emergency department patients with suspected acute coronary syndromes: a multicenter trial. J Am Coll Cardiol. 2010;56:290-299.

13. Tong CW, Madhur MS, Rzeszut AK, et al. Status of early-career academic cardiology: a global perspective. J Am Coll Cardiol. 2017;70:2290-2303. 